\title{
AN INTELLIGENT SPATIAL PROXIMITY SYSTEM USING NEUROFUZZY CLASSIFIERS AND CONTEXTUAL INFORMATION
}

\author{
F. Barouni'*, B. Moulin \\ Laval University, Computer Science Department, Pavillon Pouliot, 1065, rue de la Médecine \\ Quebec City QC G1V 0A6, Canada - foued.barouni.1@ulaval.ca, bernard.moulin@ift.ulaval.ca
}

\begin{abstract}
KEY WORDS: GIS, Decision Support, Reasoning, Artificial Intelligence, Contextual, Analysis
\end{abstract}
\begin{abstract}
:
In this paper, we propose a novel approach to reason with spatial proximity. The approach is based on contextual information and uses a neurofuzzy classifier to handle the uncertainty aspect of proximity. Neurofuzzy systems are a combination of neural networks and fuzzy systems and incorporate the advantages of both techniques. Although fuzzy systems are focused on knowledge representation, they do not allow the estimation of membership functions. Conversely, neuronal networks use powerful learning techniques but they are not able to explain how results are obtained. Neurofuzzy systems benefit from both techniques by using training data to generate membership functions and by using fuzzy rules to represent expert knowledge. Moreover, contextual information is collected from a knowledge base. The complete solution that we propose is integrated in a GIS, enhancing it with proximity reasoning. From an application perspective, the proposed approach was used in the telecommunication domain and particularly in fiber optic monitoring systems. In such systems, a user needs to qualify the distance between a fiber break and the surrounding objects of the environment to optimize the assignment of emergency crews. The neurofuzzy classifier has been used to compute the membership function parameters of the contextual information inputs using a training data set and fuzzy rules.
\end{abstract}

\section{INTRODUCTION}

One of the objectives of Human-Machine Interaction (HMI) is to provide systems that users can use in an intuitive way. In the context of qualitative spatial reasoning, the notion of proximity is one of the foundational concepts in daily human cognition studied by researchers during the last decades. Several authors proposed tools to reason about proximity and solutions which can be automated and integrated in geographic information systems (GIS). The goal is to reduce the semantic gap between quantitative data in GIS (metric distance) and qualitative data (proximity) as used by humans (Cohn and Renz, 2007). Such works used advanced qualitative techniques such as fuzzy sets and fuzzy logic as well as conceptual notions such as influence and impact areas. Empirical experiments were also conducted. However, spatial distance, on which most of these works based their solutions, is not the only factor that influences human reasoning about spatial proximity. Actually, proximity relations have two characteristics: they are context dependent and uncertain. For example, the means of transportation used to travel from Paris to London may change the traveler's perception of distance (context-dependence). When a person parks a car, she does not need to know the exact distance of the empty space between two cars (uncertainty). A suitable model of spatial proximity should consider both characteristics in order to be closer to the human apprehension of proximity.

In this paper, we propose a novel approach to reason with spatial proximity. The approach is based on contextual information and uses a neurofuzzy classifier to handle the uncertainty aspect of proximity. Neurofuzzy systems are a combination of neural networks and fuzzy systems and incorporate the advantages of both techniques. Fuzzy systems are focused on knowledge representation, but they do not allow the estimation of membership functions. Conversely, neuronal networks use powerful learning techniques but they are not able to explain how results are obtained. Neurofuzzy systems benefit from both techniques by using training data to generate membership functions and by using fuzzy rules to represent expert knowledge. Moreover, contextual information is collected from a knowledge base. The complete solution that we propose is integrated in a GIS, enhancing it with proximity reasoning capabilities. From an application perspective, the proposed approach was used in the telecommunication domain and particularly for fiber optic monitoring. In such systems, a user needs to qualify the distance between a fiber break and the surrounding objects of the environment to optimize the assignment of emergency crews. The neurofuzzy classifier (NFC) was used to compute the membership function parameters of the contextual information inputs using a training data set and fuzzy rules.

This paper is organized as follows. Section 2 discusses some related works about qualitative spatial proximity and their limitations. Section 3 presents an overview of the neurofuzzy classifier used in our approach. Section 4 presents a case study that will be used to illustrate our approach. Section 5 presents the results and section 6 discusses some research outlooks and concludes this paper.

\section{RELATED WORKS}

Reasoning with spatial proximity is a research area which has been addressed by the qualitative spatial reasoning community, adopting different perspectives such as geography, cognitive science, linguistics and others (Yao and Thill, 2007). A large number of prior works used fuzzy logic and qualitative techniques to deal with spatial proximity because it has inherent fuzziness (Robinson, 1990). While reasoning with proximity, human beings may also consider metric distances and other

* Corresponding author. This is useful to know for communication with the appropriate person in cases with more than one author. 
parameters called contextual information. In the following subsections, we present an overview of some of the works which used uncertainty techniques and contextual information or a combination of both aspects.

\subsection{Distance-based approaches}

Guesgen (2002) used fuzzy sets and associated each set with a qualitative spatial relation. The idea behind Guesgen's approach is to interpret qualitative proximity relations between spatial objects as restrictions of spatial linguistic variables such as near and far. Each linguistic variable is associated with a fuzzy set. The proximity relation is therefore represented by a membership degree of each of these fuzzy sets using a membership function. For example, the relation "the object $A$ is near to" may be interpreted by a near membership value which is associated with each near relation between object $\mathrm{A}$ and surrounding objects such as B, C and D. Generally, the nearest an object is to $\mathrm{A}$, the highest its membership value is. A software implementation used Euclidian distance between two objects to implement Guesgen's formalism. A Java-based implementation allows a user to define a "nearness" factor which will be used to specify other proximity relations. Then, the user can qualify the distance between two spatial objects using proximity relations. (Brennan and Martin, 2006) stated that most of fuzzy based proximity formalisms proposed in the literature suffer from a major shortcoming: membership functions are not clearly defined. To overcome this limitation, they used Gahegan's approach (Gahegan, 1995) who proposed a method to identify spatial proximity using three factors: the absolute distance, the relative distance between two spatial objects and the combination of both. An absolute distance may be a spatial relation such as very close, close and far. Relative distance may be a spatial relation such as closest or farthest. The combination of both absolute and relative distances was defined by Gahegan to reason about spatial relations using a fuzzy union operator. Since Gahegan did not use experimental data to validate his approach, Brennan and Martin (2006) proposed an approach to evaluate membership functions and showed how they can be combined using fuzzy logic operators. To this end, they used the absolute distance membership function $\mu_{a b s}(A, B)$ proposed by Gahegan. This function is presented in Table 1 where $A$ and $B$ are spatial objects, $\operatorname{Dist}(A, B)$ is the absolute distance between $A$ and $B$. Max is the maximum distance between all the places in the data set and it is used to normalize the value of $\operatorname{Dist}(\mathrm{A}, \mathrm{B})$. For relative distance membership function, they used the function $\mu_{\text {rel }}(A, B)$ proposed in (Worboys, 1996) and given in Table 1 where reldis $(\mathrm{A}, \mathrm{B})$ is relative distance between $\mathrm{A}$ and $\mathrm{B}$ which is calculated using the distance between $A$ and $B$ divided by the mean of distance between $A$ and each object in the data set. The result given by the Brennan and Martin's experiments demonstrated that the absolute distance and relative distance membership function can be used separately and generate linear distributions. However, combination of both metrics by union gives clustered distributions and may not be relevant for proximity reasoning whereas fuzzy intersection gives better results. The authors proposed to use this option and implemented it in a GIS. However, they neither expressed the meaning of fuzzy intersection in terms of spatial proximity,, nor did they justify the use of fuzzy logic in general. Moreover, how to apply such an approach to qualitative spatial reasoning was not obvious.

\begin{tabular}{|l|c|}
\hline Absolute Distance Metrics & $\mu_{a b s}(A, B)=1-\frac{\operatorname{Dist}(A, B)}{\operatorname{Max}}$ \\
\hline Relative Distance Metrics & $\mu_{\text {rel }}(A, B)=\frac{1}{(\operatorname{reldis}(A, B)+1)}$ \\
\hline Fuzzy Union & $\mu_{c o m b u}(A, B)=\operatorname{MAX}\left(\mu_{a b s}(A, B), \mu_{r e l}(A, B)\right)$ \\
\hline Fuzzy Intersection & $\mu_{c o m b i}(A, B)=\operatorname{MIN}\left(\mu_{a b s s}(A, B), \mu_{r e l}(A, B)\right)$ \\
\hline
\end{tabular}

Table 1: Fuzzy distance as proposed by (Brennan and Martin, 2006)

Later, (Brennan and Martin, 2012) proposed a conceptual framework to qualitatively represent spatial proximity and to enhance the capacity of spatial reasoning systems using contextual information. They consider contextual information as a key element in any model of spatial proximity. For example, a degree of proximity to an object may vary if the object is meant to be seen or reached. To reason about spatial proximity Brennan and Martin introduced the notion of impact area which is a generalization of the influence area introduced by (Kettani and Moulin, 1999). An influence area is a portion of space surrounding an object: it has an interior and exterior border such that the borders of the influence area and the border of the object have the same shape (Kettani and Moulin, 1999). Euclidean geometry has been used by Kettani and Moulin to calculate the width of an influence area. Brennan and Martin proposed a more generic approach motivated by the fact that spatial proximity is not just a metric measure. Proximity is rather context dependent. Furthermore, other spatial relations such as topological and directional relations have some unified views within the research community. Hence, they introduced the notion of impact area which involves contextual information to qualify spatial proximity. The impact area of an object takes into account both the nature of the object and its surrounding environment. Some examples in (Brennan and Martin, 2012) demonstrate how impact area is more generic than influence area, and how this notion uses contextual information in proximity analysis. Contextual information is defined as any "information collated by an expert who is expected to incorporate all relevant factors into the impact area". Figure 1 illustrates the difference between influence area and impact area for two couples of objects. Objects A1 and B1 are water tanks and objects $\mathrm{A} 2$ and $\mathrm{B} 2$ are radio towers. The distance between $\mathrm{A} 1$ and $\mathrm{B} 1$ is equal to the distance between $\mathrm{A} 2$ and $\mathrm{B} 2$. The influence area of water tank is equal to the influence area of radio towers because all objects have the same shape and size and because they are located at the same distance from each another. If we consider the functionality of the towers (range of frequency) and the surrounding Cliff, the impact areas will be different.

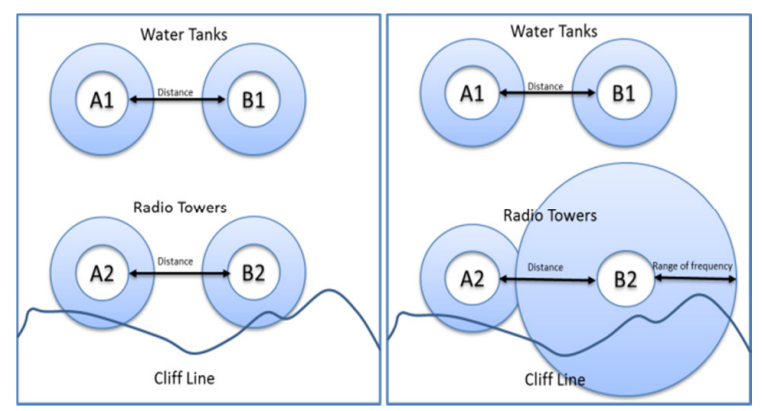

Figure 1. An example of difference between influence areas (Kettani and Moulin, 1999) and impact area (Brennan and Martin, 2012) 


\subsection{Contextual-based approaches}

(Yao and Thill, 2007) emphasized that proximity relations have two main characteristics. The first one is that proximity relation is context dependent. The authors classified context factors as 'objective' and 'subjective'. Subjective context factors may have different values according to the involved person. Examples include the navigator's familiarity with the area, his time and his budget. Objective context factors have independent values relative to the person who perceives the distance like the type of activity (run, walk), object reachability and transportation mode to name a few. The second characteristic of proximity is the uncertainty of distance measures. If a person wants to park his car, he does not need to know the exact distance between his car and the other cars around. The authors proposed a review on existing approaches based on fuzzy logic to handle the uncertainty aspect of proximity measures and they noticed that most of these works presume the form of membership functions. To overcome this limitation, Yao and Thill proposed a novel approach based on neurofuzzy techniques which allows for reasoning with spatial proximity by considering contextual information and by handling its uncertainty aspect. Neurofuzzy systems are a combination of neural networks and fuzzy systems. They incorporate the advantages of both techniques. Fuzzy systems are focused on knowledge representation while they do not support learning techniques. Conversely, neuronal networks are more powerful in learning techniques but they cannot explain how the results are obtained. Neurofuzzy systems, take advantage of both techniques by using training data instead of preset membership functions and by using fuzzy rules fto represent expert knowledge.

The contextual factors used in Yao and Thill's approach (objective and subjective) take the form of crisp inputs. They are fuzzyfied using membership functions (which can be predetermined by an expert). Fuzzyfied outputs are generated as consequences of applying all fuzzy rules. The final output (either fuzzy or crisp) is calculated through defuzzification using the weighted average of fuzzyfied outputs. Yao and Thill used ANFIS (Adaptive NeuroFuzzy Inference System) to implement and validate their approach. ANFIS is a TakagiSugeno fuzzy inference system (Yao and Thill, 2007). It uses a back propagation algorithm to train the fuzzy neural network. This algorithm computes the error between the training data and the neural network output and uses the error to adjust the rules' weights. The experimental results demonstrated that a neurofuzzy approach gives higher prediction accuracy when training data and testing data are compared. Finally, the proposed approach allowed resolving the problem of using presumed membership functions reported by the authors and by (Brennan and Martin, 2006)

\subsection{Discussion}

Qualitative reasoning with spatial proximity has been addressed by the above works which tried to design solutions that can be automated and easily integrated in a GIS and which reduce the semantic gap between quantitative data in a GIS (distance) and qualitative data (proximity) as perceived by humans. However, the distance-based approaches are not necessarily the only factor that influences human cognitive apprehension of spatial proximity. A suitable model of spatial proximity should acknowledge other factors such as contextual information as proposed by (Brennan and Martin, 2012). The latter work suffers from two main shortcomings: first it only allows to reason about nearness. There is no clear definition of other proximity relations such as "far", "close to" and so on. Therefore, it difficult to relate their definition of impact area with different proximity relations used by humans and in GIS solutions. Second, the definition of impact area seems to be very domain specific. Examples provided by the authors do not clearly explain how and from where contextual information is gathered and how contextual information can impact the spatial proximity. Therefore, implementing this work in a generic GIS is quite challenging. (Yao and Thill, 2007) proposed an innovative solution to reason about proximity by using contextual information to handle the cognitive aspect and neurofuzzy techniques to handle the qualitative aspect of spatial proximity. Although Yao and Thill's approach may be suitable for qualitative spatial reasoning, it suffers from several drawbacks. The neurofuzzy system used for the implementation and experiments is ANFIS which is an approximation system (Nauck and Kruse, 1999). ANFIS' output is a crisp value which does not help in "classifying" the spatial proximity. Usually, given a number of context factors, a user/agent tries to answer to the following question: what is the proximity relation between object $A$ and object $B$ ? Possible answers may be very near, near, far or very far. We believe that qualitative proximity is a classification problem rather than an approximation problem. Furthermore, Yao and Thill proposed a general architecture to implement their solution and conducted experiments to prove its relevance. However, teh solution was not integrated in a GIS. In the next sections, we propose a new framework to reason about qualitative proximity with the following features: first, a neurofuzzy classifier is used instead of ANFIS in order to handle the uncertainty aspect of proximity. Second, we integrate the proposed solution in a GIS to enhance its qualitative proximity reasoning capabilities. Figure 2 illustrates the general architecture of our approach. A user specifies contextual information and fuzzy rules to generate training data set which is used by the NFC to train the fuzzy inference system. An overview of the NFC structure used in our approach is presented in the following section.

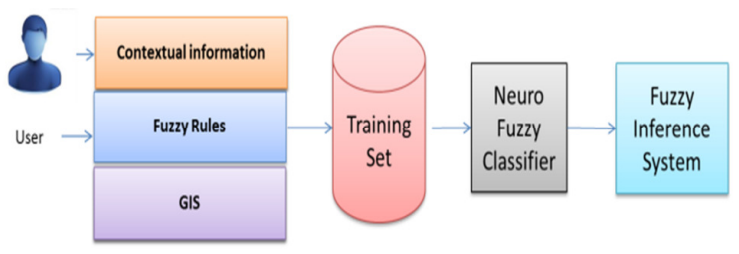

Figure 2. An overview of the proposed approach

\section{NEUROFUZZY CLASSIFIER STRUCTURE}

The structure of a neuro-fuzzy system is similar to a multilayer neural network. In general, a neuro-fuzzy system has one input layer, one output layer, and three hidden layers (Negnevitsky, 2011). In the neuro-fuzzy classification systems, the feature space is partitioned into multiple fuzzy subspaces which are managed by fuzzy rules. Rules are represented by a network structure and their parameters (weights) are optimized using learning techniques.

A fuzzy classification rule $R i$; establishes the relation between the input feature spaces and classes (output). It is defined as follows:

$$
\begin{aligned}
& R_{i}: \text { if } x_{c 1} \text { is } S_{i 1} \text { and } \ldots \ldots x_{c j} \text { is } S_{i j} \\
& \quad \text { and } x_{c n} \text { is } S_{i n} \text { then output class is } C_{k}
\end{aligned}
$$


Where $x_{c j}$ is the $j^{\text {th }}$ input variable of the $c^{\text {th }}$ sample; $S_{i j}$ denotes the fuzzy set of the $j^{\text {th }}$ feature in the $i^{\text {th }}$ rule; and $C_{k}$ represents the $k^{\text {th }}$ label of class. $S_{i j}$ is associated with the suitable membership function (Sun and Jang 1993).

Using fuzzy rules allows for splitting the feature space into multiple fuzzy subspaces. These rules can be represented by a neural network. Figure 3 depicts an example of a space partition of two inputs $\mathrm{x} 1$ and $\mathrm{x} 2$. Each input (feature) has three fuzzy sets described by linguistic variables. Hence, we have nine fuzzy rules overall. A neurofuzzy classifier is a multilayer network with five layers. The first layer is the input layer (features). The last layer is the output layer (classes). The other layers are defined as follows:

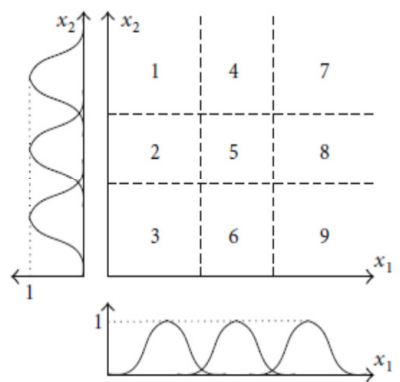

Figure 3. partition of the feature space (Sun and Jang, 1993).

Membership layer: each input is identified using fuzzy sets and each fuzzy set is associated with a linguistic variable. Fuzzy sets are represented by membership functions. According to (Cetişli and Barkana, 2010) bell-shaped functions are the most used functions in neurofuzzy classifiers since this function has fewer parameters and smoother partial derivatives as parameters. This function is used for this layer and is defined as follows:

$$
\mu_{i j}\left(x_{c j}\right)=\exp \left(-\frac{\left(x_{c j}-c_{i j}\right)^{2}}{2 \sigma^{2} i j}\right)
$$

Where $\mu_{i j}\left(x_{c j}\right)$ is the membership grade of $i_{\mathrm{th}}$ rule and $j_{\mathrm{th}}$ feature; $x_{c j}$ represents the $c_{\mathrm{th}}$ sample and $j_{\mathrm{th}}$ feature; $c_{i j}$ and $\sigma_{i j}$ are the center and the width of bell shaped function, respectively. The membership functions of input variables $\mathrm{x}_{1}$ and $x_{2}$ of Figure 3 are examples of bell shaped function.

Fuzzification layer: each node in this layer is a fuzzy rule. The antecedent of the fuzzy rule is a fuzzy set. The output is a singleton membership function. The fuzzy rule premises become weights for the rule neurons of this layer (Gliwa and Birsky, 2011). The conclusion of a rule is a connection from the rule neuron to the next layer. Each node in this layer has an activation function which corresponds to the degree of fulfillment of the fuzzy rule for the $x_{c j}$ sample. The activation function $a_{i c}$ of a fuzzy rule is defined as follows:

$$
a_{i c}=\prod_{j=1}^{n} \mu_{i j}\left(x_{c j}\right)
$$

Where $n$ is the total number of features.

Defuzzification layer: in this layer, each rule affects each class according to their weights. The more a rule impacts a class, the bigger the weight between that rule output and the specific class is. Otherwise, the class weights are small. The weighted output for a given sample $\mathrm{x}$ that belongs to a class $\mathrm{k}$ is computed as follows:

$$
\beta_{c k}=\sum_{i=1}^{Z} a_{i c} w_{i k}
$$

Where $w_{i k}$ denotes the degree of belonging to the $k_{\mathrm{th}}$ class that is controlled by the $i_{\text {th }}$ rule and $\mathrm{Z}$ represents the number of rules (Do and Chen, 2013).

Normalization layer: in some cases, the summation of the weighted sum can be larger than 1 . Therefore, this sum should be normalized using the following formula:

$$
N_{c k}=\frac{\beta_{c k}}{\sum_{h=1}^{K} \beta_{c h}}
$$

Where $N_{c k}$ is the normalized value of the $c_{\text {th }}$ sample that belongs to the $k_{\mathrm{th}}$ class and $K$ is the number of classes.

Finally, the output class $C_{c}$ is the maximum of normalized values given by the normalization layer:

$$
C_{c}=\max _{k=1,2, \ldots, k, k}\left\{N_{c k}\right\}
$$

Figure 4 depicts a neurofuzzy classifier network with the different layers and with two input features and 3 output classes.

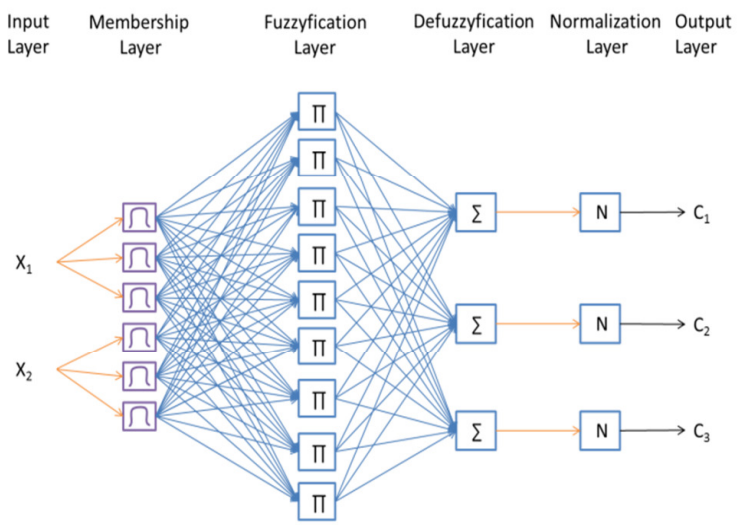

Figure 4. A neurofuzzy classifier (Cetişli and Barkana, 2010)

\section{CASE STUDY}

We use a case study to illustrate the relevance of our approach and for experimentation purposes. A Remote Fiber Test System (RFTS) is a real time acquisition system which is designed to monitor fiber optic networks. It is widely used in the Telecommunications industry to optimize the service level agreement (SLA) between service providers and consumers. It can also be used in the Oil and Gas industry to monitor pipelines. Fibers can be installed along the pipelines and the occurrence of a fiber fault means the occurrence of a pipeline damage event.

The RFTS is based on the Optical Time Domain Reflectometer (OTDR) technology. OTDRs are commonly used to characterize the loss and length of fibers as they go from initial manufacturing, through to cabling, warehousing while wound on a drum, installation and then splicing. OTDRs are also commonly used for fault finding on installed systems. In this case, reference to the installation OTDR trace is very useful, to determine where changes have occurred (Wikipedia, 2014). 
Figure 5 depicts the accordance between the OTDR trace and the fiber optic characteristics.

An RFTS is a distributed system where Remote Terminal Units (RTU) are geographically deployed and continuously run to monitor the fiber status using OTDRs. When a fiber fault occurs, it is detected by an RTU and reported to a central server which maps the fault in a GIS. Figure 6 depicts the general structure of a remote fiber test system.

A user needs to evaluate the distance between the fault location and several locations such as: crew location, location of critical sites where service level agreement must be fully respected (bank, broadcasting channel building) and asset locations (splice point, transmission equipment and so on). Hence, the notion of proximity is very important in such applications and it helps users make decisions. In the following section, we present an overview of the NFC which we use to build such a spatial proximity framework.

\section{OTDR Trace Information}
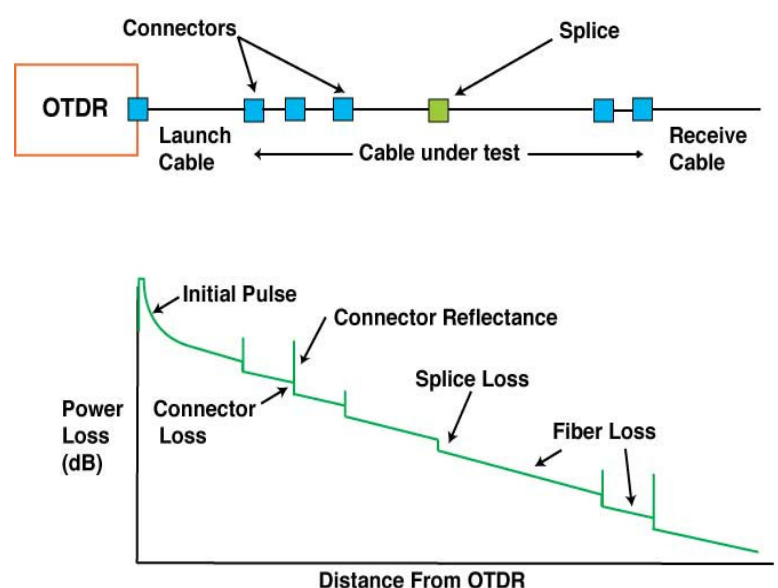

Figure 5. OTDR Trace Information (Thefoa.org, 2014)

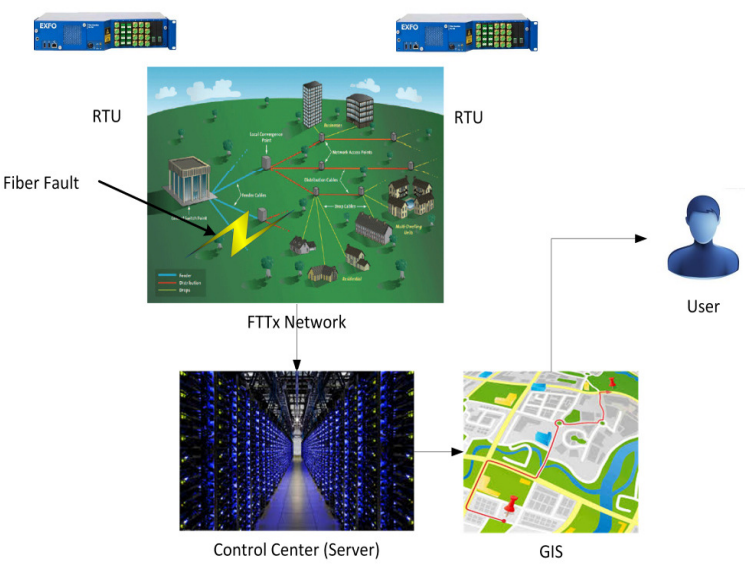

Figure 6. General architecture of RFTS

\section{EXPERIMENTS}

\subsection{Training the neurofuzzy classifier}

One of the advantages of using a neurofuzzy classifier is that membership functions and rules can be learned from data sets. If $S_{Z \times n}$ and $C_{Z \times n}$ are the sigma and the center values of the bell membership function; and $W_{Z \times K}$ is the weight matrix of connection from fuzzification to defuzzification layers; then $O=\left\{S_{Z \times n}, C_{Z \times n}, W_{Z \times K}\right\}$ is the set of parameters that will be optimized by the learning algorithm.

Several training algorithms have been proposed for neurofuzzy classifiers (Do and Chen, 2013). However, the scale conjugate gradient (SCG) is one of the most efficient algorithms with less errors and high efficiency. This algorithm was enhanced and implemented in a software package by (Cetişli and Barkana, 2010). We use this algorithm for our data set training. A detailed overview about this training algorithm can be found in (Cetişli and Barkana, 2010).

A Matlab implementation of the NFC has been developed by (Cetişli and Barkana, 2010). It first trains the NFC, and then it generates the fuzzy inference system parameters that are used by the qualitative proximity reasoning engine. In this section, we present how data sets are prepared to train the NFC and we present the obtained results.

\subsection{Input selection and data set}

We use Yao and Thill's definition (2007) of contextual information to select the NFC inputs. Objective and subjective contextual informations are used respectively when variables are independent from the person who perceives the distance, and when variables are dependent on the person who perceives the distance. We use Euclidian distance as the objective input. Road traffic and user's familiarity with the area are used as subjective contextual information. Three classes are used for the proximity output (short, medium and far). The following tables 2 and 3 detail the NFC inputs and outputs:

\begin{tabular}{|l|l|l|}
\hline Variable name & Fuzzy sets & Range \\
\hline $\begin{array}{l}\text { Euclidian } \\
\text { distance }\end{array}$ & Short, medium, far & $0-10000 \mathrm{KM}$ \\
\hline Traffic & Light, medium, heavy & $0-1$ \\
\hline $\begin{array}{l}\text { User familiarity } \\
\text { with the site }\end{array}$ & Week, average, good & $0-1$ \\
\hline
\end{tabular}

Table 2: NFC features (inputs)

\begin{tabular}{|l|l|}
\hline Class & Value \\
\hline Very Short & 1 \\
\hline Short & 2 \\
\hline Medium & \\
\hline Far & 3 \\
\hline
\end{tabular}

Table 3: NFC Classes (outputs)

It is worth noting that the Euclidian distance is used for simplification purposes. Normally, a distance between two geographic features is computed using a path planning software. A data set with 300 samples has been prepared using locations in Quebec City. 150 samples are used to train the NFC and 150 samples are used to test the trained NFC. To generate the different classes (outputs) in the sample data set, we used a 
fuzzy inference system (FIS) with 13 rules specified thanks to the user's experience and illustrated by Figure 7 .

Each feature is associated with three fuzzy sets which are represented by a bell shaped membership function. The FIS uses a Sugeno type system to calculate the weighted sum of the fuzzy rules output for each sample vector. The FIS gives a crisp output with a float number which is not suitable to make a decision about which class the proximity belongs to. Therefore, we apply a "round function" to generate the number that converts it to the final class. An algorithm to automate data generation is described by the pseudo code in Table 4 .

The data set has been created using a specific area which is delimited using a square. All the distances are computed between geographic objects that belong to this specific area. Therefore, the training results will be valid only for this specific area. If the area changes, the NFC training must be repeated for the new area.

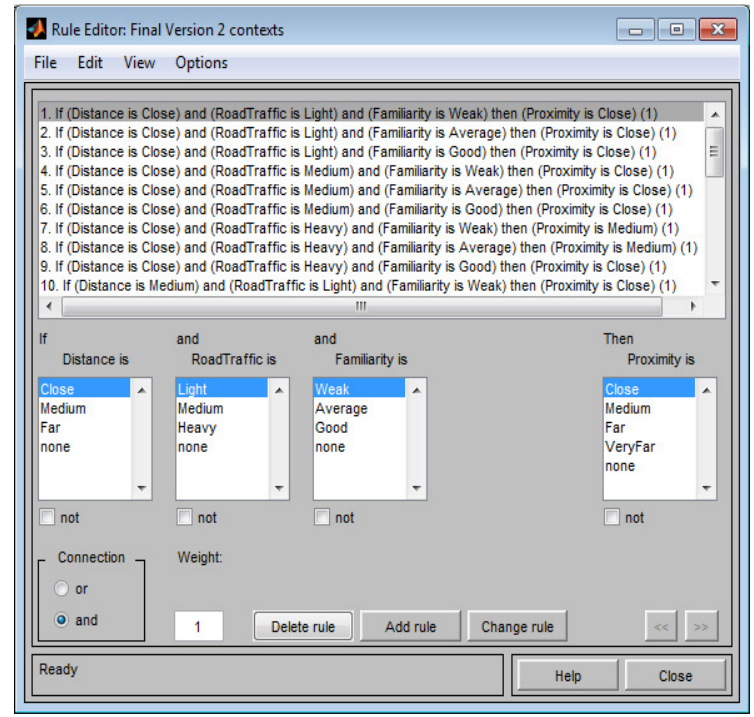

Figure 7. Fuzzy rules used to prepare the data set

Generate Data Set $(\mathrm{V}, \mathrm{O})$

$\mathrm{V}$ : vector of inputs

O: output of the FIS

1. Get all the geographic objects inside the training area.

2. Compute the Euclidian distance between the objects.

3. For each distance between two geographic objects,

4. Assign two contextual information

5. Save in the Sample Data Set

6. End For

7. Load Sample Data

$x_{c 1}$

8. For each vector feature from the $\mathrm{V}=x_{c 2}$ in the data set,

9. Calculate the output of the fuzzy rules.

10. Calculate the FIS output of the specific vector V

11. The output class = Ceil the FIS output.

12. End For

Table 4: NFC data set training preparation

\section{RESULTS}

Now that our data set is ready, we use it to train the NFC. The root mean square error (RMSE) is used to evaluate the error between the NFC output and the testing output. It converges after 55 epochs and remains stable at 0.229963 (Figure 8). This means that $77 \%$ of the testing inputs have been successfully classified by the NFC. The classifier generated a new set of features with new fuzzy sets and new membership functions. For example, the distance feature in the data set used to train the algorithm had three fuzzy sets: short, medium and far. The new distance feature has four fuzzy sets named as follows: close, average, far and very far. Obviously, these names are chosen by an expert who intuitively associates the linguistic variables with different features proposed by the NFC.

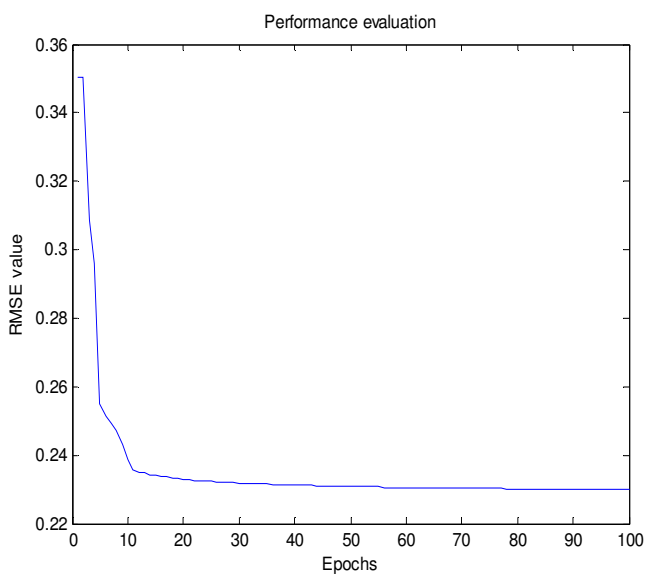

Figure 8. NFC training Performance for 3 features and 4 classes

The same logic applies to other features (road traffic and user's familiarity with the region). Figures 9 (a, b, c, d, e, f) illustrate the fuzzy sets of each input feature as proposed by the user to generate the data set and the fuzzy sets of each input feature as classified by the NFC. These outputs are only valid for the geographic area in which the data set has been defined. If the area changes, these membership functions are no longer valid and the system should be trained again.

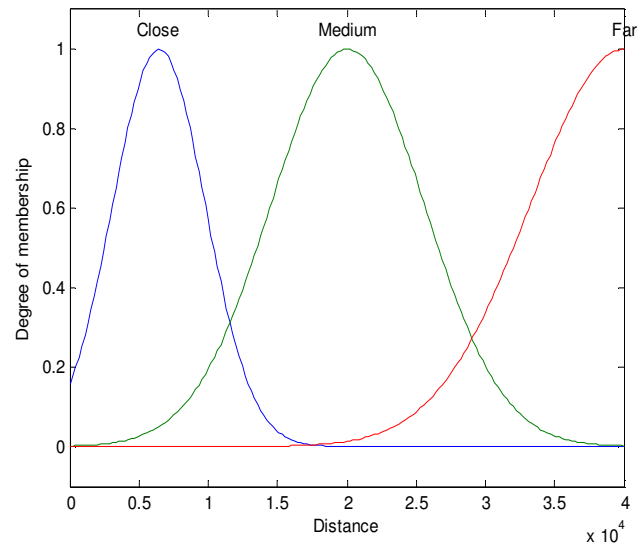

(a) distance feature as defined by user to train the NFC 


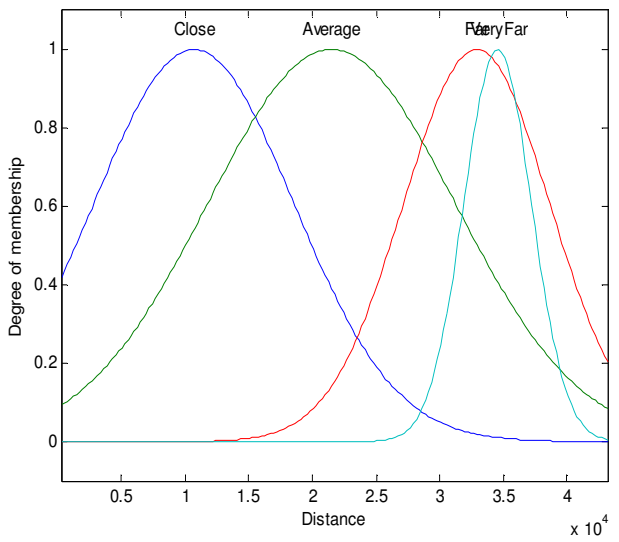

(b) distance feature after the NFC training

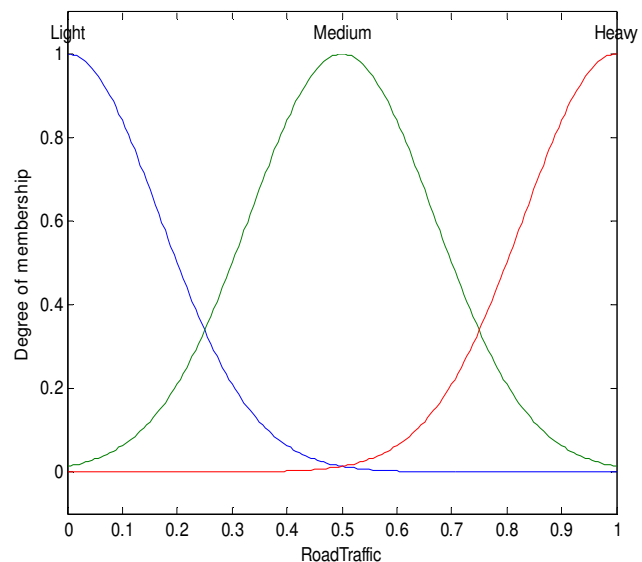

(c) road traffic feature as defined by user to train the NFC

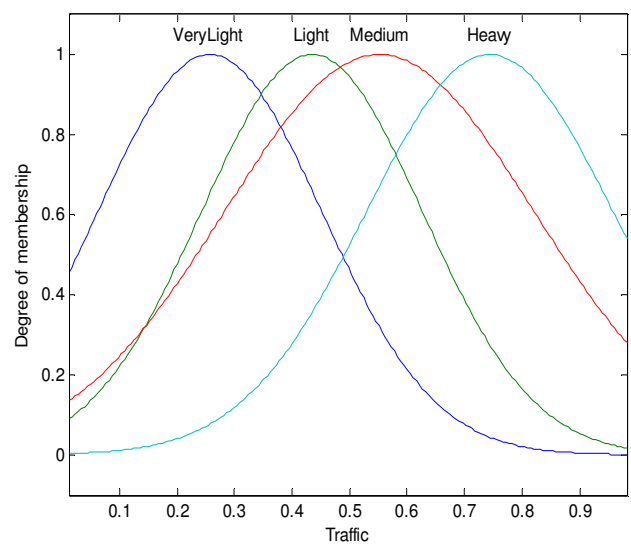

(d) road traffic feature after the NFC training

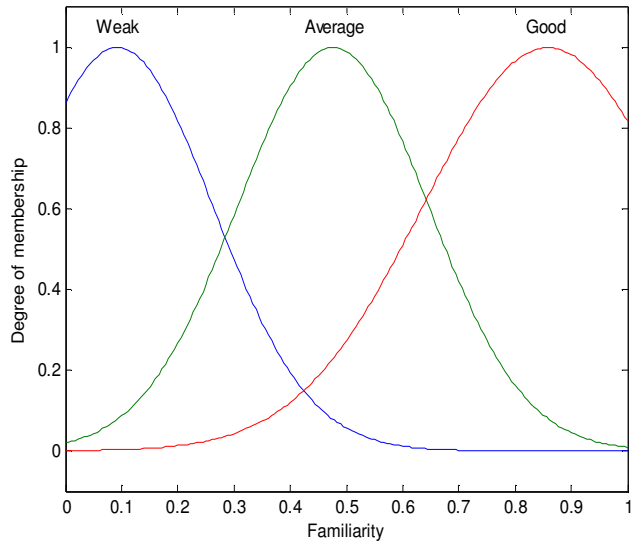

(e) User's familiarity with area feature as defined by user to train the NFC

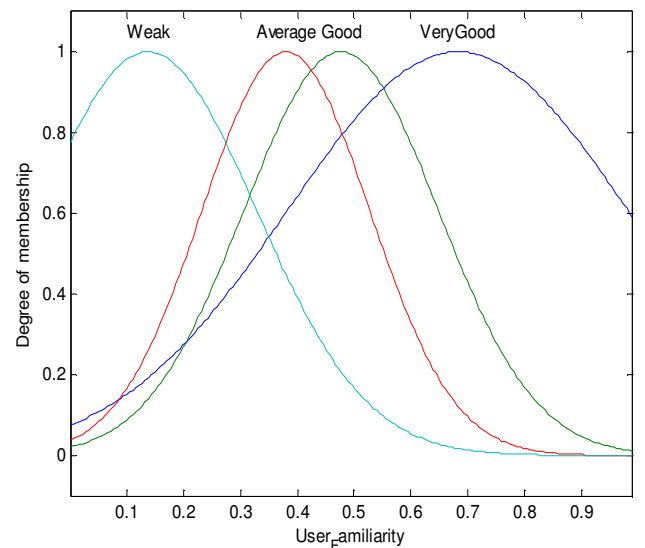

(f) User's familiarity with area feature after the NFC training

Figure 9. NFC outputs

\section{A QUALITATIVE PROXIMITY TOOL: ARCHITECTURE AND IMPLEMENTATION}

The main goal of using NFC is to generate membership functions for the fuzzy inference system based on expert's knowledge. A software tool has been developed to integrate the NFC output in a qualitative proximity reasoning tool which is integrated to a GIS.

\subsection{Architecture}

The general architecture of the tool is depicted in Figure 10. The NFC is a Matlab based program which uses the data set to generate the fuzzy membership functions and the fuzzy rules. These outputs are used by a fuzzy inference system (FIS) managed by the jFuzzyLogic module. jFuzzyLogic is an open source fuzzy logic library implementing industry standards to simplify the development of fuzzy systems. It is a java package which uses FIS files to reason with fuzzy rules. The Java Topology Suite (JTS) is a software package which is used to handle the spatial calculus of geographic features which are stored in the database (PostGIS).

\subsection{Workflow}

Training the system using the NeuroFuzzy classifier is the very first step in using the developed tool. A user should select a 
specific geographic area where contextual information is available (distance, traffic and so on) and then generate the data set that will be used to train the classifier. When a fiber fault occurs in the field, a distance between the fault location and the reference object is calculated. The reference object can be selected by the user using various criteria:

- The distance between the fiber fault location and the RTU that is monitoring the fiber status;

- The distance between the fiber fault location and the central office location. The central office is a corporate building where crews are waiting to be assigned for a fiber inspection;

- The distance between the fiber fault location and telecommunication assets such as splice points, patch panels, transmission equipment, etc.

- The distance between the fiber fault location and critical locations where service level agreement with customers must be fully respected such as banks, military locations etc.

A user shall select the distance and other contextual informations to qualify the distance. The software output is a qualitative proximity measure expressed using a linguistic variable (close, far from etc.). Such a tool can be integrated in any decision based system where the concept of quality of service is very important and the notion of proximity to an event plays a key role in the user's decision.

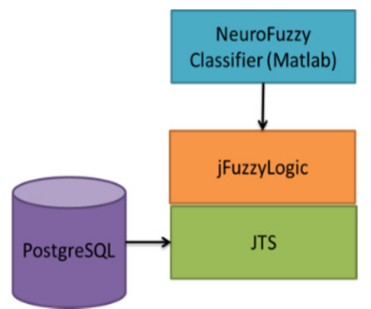

Figure 10. Qualitative proximity software architecture

\section{DISCUSSION AND CONCLUSION}

Qualitative reasoning about spatial proximity is not only a distance issue. Other factors can influence the user's perception about proximity relation between two locations. In addition, the proximity relation is uncertain and fuzzy. A human being does not need to know the exact metric values of distances in his daily's life.

In this paper, we considered the former aspects in designing a solution for qualitative spatial proximity reasoning tool. Such a solution is integrated in a GIS and can be used in various application domains. Some improvements remain to be made in our solution. For example, it would be interesting to enhance the human machine interaction model so that a user can pick up locations and select contextual information and get the proximity relation. Also, the contextual information is currently specified from a simplified database. In the future, it would be interesting to gather such information from a knowledge base.

\section{REFERENCES}

Brennan, J., Martin, E., 2006. Membership functions for spatial proximity. In: Sattar, A., Kang, B.H. (Eds.), AI-2006. Lecture Notes in Artificial Intelligence, vol. 4304. Springer Verlag, Berlin, pp. 942-949.
Brennan, J., Martin, E., 2012. Spatial proximity is more than just a distance measure. Int. J. Hum.-Comput. Stud. Vol. 70(1), pp. 88-106.

Cetişli, B., Barkana, A., 2010. Speeding up the scaled conjugate gradient algorithm and its application in neuro-fuzzy classifier training. Soft Computing, 14(4), 365-378.

Cohn, A.G, Renz J., 2007. Qualitative Spatial Representation and Reasoning, In: Frank van Harmelen, Vladimir Lifschitz and Bruce Porter, Editor(s), Foundations of Artificial Intelligence, Elsevier, vol. 3, pp. 551-596.

Do, Q. H., Chen, J. F., 2013. A Neuro-Fuzzy Approach in the Classification of Students' Academic Performance, Computational Intelligence and Neuroscience vol. 2013 Article ID 179097.

Gahegan, M., 1995. Proximity Operators for Qualitative Spatial Reasoning. In COSIT '95 Proceedings: Spatial Information Theory: A Theoretical Basis for GIS, 31-44, edited by A. U. Frank and W. Kuhn. Berlin, Germany: Springer-Verlag.

Gliwa, B., Byrski, A., 2011. Hybrid Neuro-Fuzzy Classifier Based on NEFCLASS Model. Computer Science. pp. 115-135. ISSN 1508-2806.

Guesgen, H. W., 2002. Reasoning About Distance Based on Fuzzy Sets. Applied Intelligence, vol. 17, pp. 265-70.

Kettani, D., Moulin, B., 1999. A spatial model based on the notion of spatial conceptual map and of object's influence areas. In: Mark, D.M., Freksa, C. (Eds.), Spatial Information Theory Cognitive and Computational Foundations of Geographic Information Science. LCNS, vol. 1661. Springer Verlag, Berlin, pp. 401-416.

Nauck, D., Kruse, R., 1999. Neuro-fuzzy systems for approximation functions, Fuzzy sets and systems, vol. 101, pp. 261-271.

Negnevitsky, M., 2011, Artificial Intelligence: A Guide to Intelligent Systems. Addison-Wesley Longman Publishing Co., Inc. Boston, MA, USA

Robinson, V. B., 1990. Interactive Machine Acquisition of a Fuzzy Spatial Relation. Computers and Geosciences, vol. 16(6), pp. 857-72.

Sun, C.-T., Jang, J.-S., 1993. A neuro-fuzzy classifier and its applications. Fuzzy Systems, vol. 1, pp. 94-98.

Thefoa.org, 2014, www.thefoa.org. (April 29, 2014).

Wikipedia, 2014. www.wikipedia.org. (April 29, 2014).

Worboys, M. F., 1996. Metrics and Topologies for Geographic Space. In Advances in Geographic Information Systems Research II: Proceedings of the International Symposium on Spatial Data Handling, 365-76, edited by M. J. Kraak and M. Molenaar.

Yao, X., Thill, J.-C., 2007. Neurofuzzy Modeling of ContextContingent Proximity Relations. Geographical Analysis, vol. 39, pp. 169-194. 